\title{
Histological study of the biodynamics of iron oxide nanoparticles with different diameters
}

\author{
This article was published in the following Dove Press journal: \\ International Journal of Nanomedicine \\ I August 201 | \\ Number of times this article has been viewed
}

\author{
Keiko Tsuchiya' \\ Norihisa Nitta' \\ Akinaga Sonoda' \\ Ayumi Nitta-Seko' \\ Shinichi Ohta' \\ Hideji Otani' \\ Masashi Takahashi' \\ Kiyoshi Murata' \\ Katsutoshi Murase ${ }^{2}$ \\ Satoshi Nohara ${ }^{2}$ \\ Kenichi Mukaisho ${ }^{3}$ \\ 'Department of Radiology, \\ Shiga University of Medical Science, \\ Otsu, Shiga, ${ }^{2}$ The Nagoya Research \\ Laboratory, Meito Sangyo Co, \\ Ltd, Kiyosu, Aichi, ${ }^{3}$ Department of \\ Pathology, Shiga University of Medical \\ Science, Otsu, Shiga, Japan
}

Correspondence: Keiko Tsuchiya Department of Radiology, Shiga University of Medical Science, Setatsukinowa-cho, Otsu,

Shiga 520-2192, Japan

Tel +81775482288

Fax +8I 775440986

Email keikot@belle.shiga-med ac.jp

\begin{abstract}
The biodynamics of ultrasmall and small superparamagnetic iron oxide (USPIO and SPIO, respectively) particles that were injected intraperitoneally into $36 \mathrm{C} 57 \mathrm{BL} / 6$ mice were investigated chronologically. Their distribution was studied histologically at six time points by measuring iron-positive areas $\left(\mu \mathrm{m}^{2}\right)$ in organ sections stained with Prussian blue. The uptake of the differently sized particles was also compared by cultured murine macrophages (J774.1). Iron-positive areas in the liver were significantly larger in the mice injected with USPIO than those injected with SPIO at the first three time points $(P<0.05)$. The amount of USPIO in the lung parenchyma around the airway was larger than that of SPIO at four time points $(P<0.05)$; distribution to the lymph nodes was not significantly different. The amount of iron was significantly larger in SPIO- than USPIO-treated cultured cells $(P<0.05)$. In conclusion, it is suggested that intra peritoneally injected USPIO particles could be used more quickly than SPIO to make Kupffer images of the liver and that both agents could help get lymph node images of similar quality.
\end{abstract}

Keywords: USPIO, SPIO, contrast agent, biodistribution, histology

\section{Introduction}

The uptake of superparamagnetic iron oxide (SPIO) particles by cells of the mononuclear phagocytic system (MPS) results in hepatic, splenic, bone marrow, and nodal iron accumulation. ${ }^{1,2}$ The selective accumulation of these particles by the MPS is exploited at organ-specific magnetic resonance imaging (MRI). ${ }^{3-10}$ The biodynamics of iron oxide nanoparticles vary depending on particle diameter. Unlike SPIO preparations comprised of large particles and magnetite microspheres (mean particle diameter, $72 \mathrm{~nm}$ and 1-5 $\mu \mathrm{m}$, respectively), ultrasmall superparamagnetic iron oxide (USPIO) particles (mean diameter, $18 \mathrm{~nm}$ ) are not immediately recognized by the hepatic and splenic MPS. ${ }^{11,12}$ The resultant prolongation of its intravascular half-life, together with its inherent $\mathrm{T} 1$ shortening properties, render USPIO a useful magnetic resonance angiography blood-pool agent. ${ }^{13,14}$ In contrast to large-particle superparamagnetic agents, the size of USPIO particles allows the extravasation of USPIO through capillary pores, the diameters of which range from 5 to $100 \mathrm{~nm}$, and this capillary permeability facilitates the uptake of USPIO in MPS cells throughout the body. ${ }^{15}$

Although information on the biodynamics of iron oxide nanoparticles of different diameters is important for various organ-specific MRI studies, to date this issue has not been addressed histologically. Therefore, a comparative histological study of the sequential biodynamics of USPIO and SPIO was performed. 


\section{Material and methods}

Shiga University of Medical Science's animal experimentation committee approved all experimental protocols and all experiments were conducted in accordance with the animal care guidelines of the university.

\section{Iron oxide nanoparticles}

Two kinds of iron oxide were studied: USPIO (Meito Sangyo Co, Ltd, Kiyosu, Aichi, Japan) and SPIO (Resovist ${ }^{\circledR}$; I'rom Pharmaceutical Co, Ltd, Shinagawa-ku, Tokyo, Japan). The mean particle diameters and iron concentrations of USPIO and SPIO were $30 \mathrm{~nm}$ and $56 \mathrm{mg} / \mathrm{mL}$ and $57 \mathrm{~nm}$ and $28 \mathrm{mg} / \mathrm{mL}$, respectively. Average diameter of the magnetic core was the same $(5 \mathrm{~nm})$ in both particle types. The iron oxide core of both particle types was coated with dextran; their magnetic susceptibility was almost the same (about $0.027 \mathrm{cgs}$ ).

\section{In vivo study}

USPIO or SPIO was injected intraperitoneally ( $500 \mu \mathrm{mol} \mathrm{Fe} / \mathrm{kg})$ into 8-week-old female C57BL/6 mice (Japan SLC, Inc, Tokyo, Japan), each weighing approximately $30 \mathrm{~g}$. Groups of three mice each were treated with USPIO or SPIO and sacrificed at time points of 30 minutes and 1, 3, 12, 24, and 48 hours afterward; two mice were the controls. Organs to be studied were fixed in 10\% paraformaldehyde and examined histologically.

\section{Histological examination}

The mice were sacrificed at the indicated time points and their organs were removed for histological study. Sections of the right lobe of the liver were cut in the axial plane; splenic sections were also cut in the axial plane and included the hilum. Lung sections were cut in the coronal plane and included the hilum. Sections from the heart, great vessels, gastrointestinal tract, and kidney were cut in the coronal plane. Sections ( $4 \mu \mathrm{m}$ in thickness) of paraffin-embedded organs were stained with Prussian blue to identify the accumulation of iron oxide. Macrophages were immunohistochemically stained with F4/80. Areas stained with Prussian blue and F4/80 were compared. For quantitative assessment, Prussian-blue-stained areas $\left(\mu \mathrm{m}^{2}\right)$ in a single field of view (magnification $\times 200$ ) of three different lesions for each histological section were measured using Image-Pro ${ }^{\circledR}$ Plus software (Media Cybernetics, Bethesda, MD) and average values were calculated.

\section{In vitro study}

To compare phagocytosis of USPIO and SPIO by cultured cells murine macrophage cell line J774.1 (RIKEN Cell Bank,
Wako, Saitama, Japan) was used, which is widely used in research on macrophages. ${ }^{16-18}$ Cells $\left(5 \times 10^{4}\right)$ were seeded in 12-well multiple well culture plates (Costar ${ }^{\circledR}$; Corning Inc, Corning, NY) and grown at $37^{\circ} \mathrm{C}$ for 24 hours in $1 \mathrm{~mL}$ of growth medium (RPMI-1640; Nacalai Tesque, Inc, Kyoto, Japan) supplemented with $10 \%$ (v/v) fetal bovine serum (FBS) (Invitrogen Ltd, Carlsbad, CA) and 1\% (w/v) penicillin and streptomycin solution (Nacalai Tesque, Inc). After 24 hours, the medium was replaced, USPIO or SPIO was added (10 $\mu \mathrm{g}: 100 \mu \mathrm{L})$ and the plates were incubated for 1 hour for cell labeling. After this, the medium was again replaced and the wells then incubated for 24 hours. To identify intracellular iron oxide accumulation, the cells were stained with Prussian blue. The amount of iron contained in cell lysates by atomic absorption photometry (AA-6800; Shimadzu Co, Kyoto, Japan) was measured. Cell labeling was as described in a previous study. ${ }^{19}$

\section{Statistical analysis}

Statistical analysis was carried out using Dr SPSS II for Windows (SPSS Japan Inc, Tokyo, Japan). Differences in the uptake of USPIO and SPIO in murine tissues and the uptake of iron by cultured cells were determined with the one-tailed Student's $t$-test. A $P$-value $<0.05$ was considered statistically significant.

\section{Results}

\section{Quantitative histological analysis}

Quantitative analysis based on the measurement of Prussianblue-stained areas revealed that iron-positive areas in the liver were larger in the mice injected with USPIO than those injected with SPIO at almost all time points except the last one ( 828 vs $0 \mu \mathrm{m}^{2}$ at 30 minutes, 1299 vs $297 \mu \mathrm{m}^{2}$ at 1 hour, 3647 vs $853 \mu \mathrm{m}^{2}$ at 3 hours, 2256 vs $1497 \mu \mathrm{m}^{2}$ at 12 hours, 1885 vs $1168 \mu \mathrm{m}^{2}$ at 24 hours, and 1948 vs $2483 \mu \mathrm{m}^{2}$ at 48 hours), as shown in Figures 1 and 2. The difference was significant at 30 minutes, 1 , and 3 hours $(P<0.05)$. USPIO was quickly distributed throughout the liver; its distribution increased until 3 hours postinjection and decreased thereafter. On the other hand, the distribution of SPIO occurred more slowly and increased over a longer period of time. Unlike USPIO, SPIO was not detected in the liver at 30 minutes. Iron-positive areas at the hepatic sinusoid corresponding to the area harboring Kupffer cells coincided with macrophage-positive F4/80-stained areas, as shown in Figure 3.

In lung specimens, a few USPIO and SPIO particles were observed early; they were located in the parenchyma around 
A

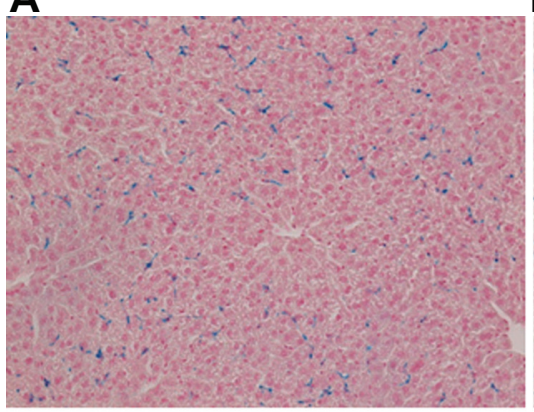

D

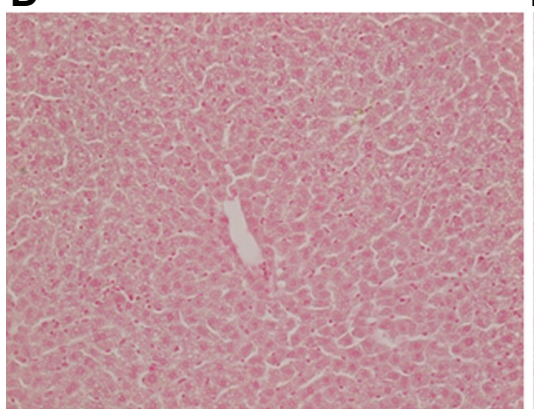

B

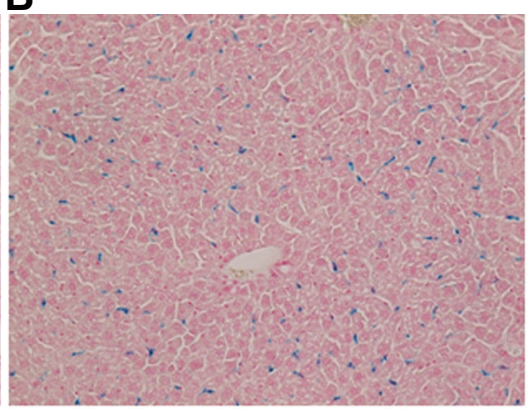

E

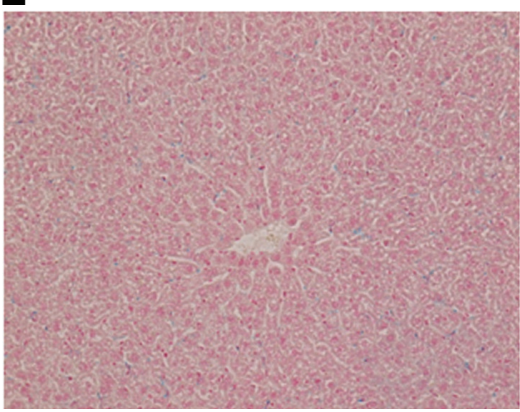

C

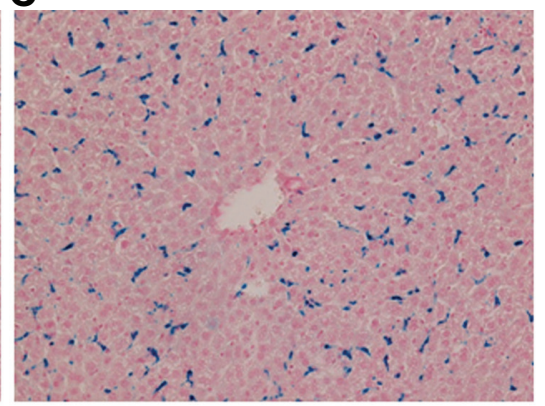

$\mathbf{F}$

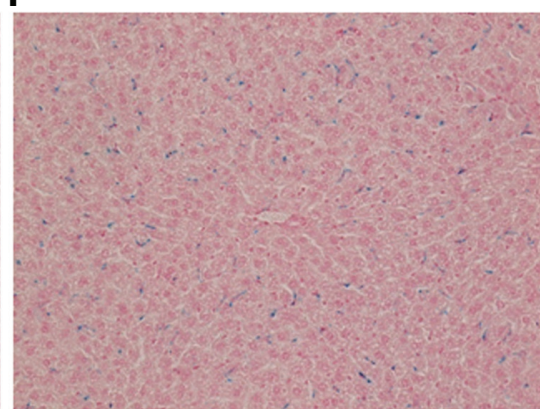

Figure I Histological study of mouse liver specimens stained with Prussian blue (magnification $\times 200)$. (A), (B), (C): 30 minutes, I, and 3 hours, respectively, after intraperitoneal (IP) injection of ultrasmall superparamagnetic iron oxide. (D), (E), (F): 30 minutes, I, and 3 hours, respectively, after IP injection of small superparamagnetic iron oxide.

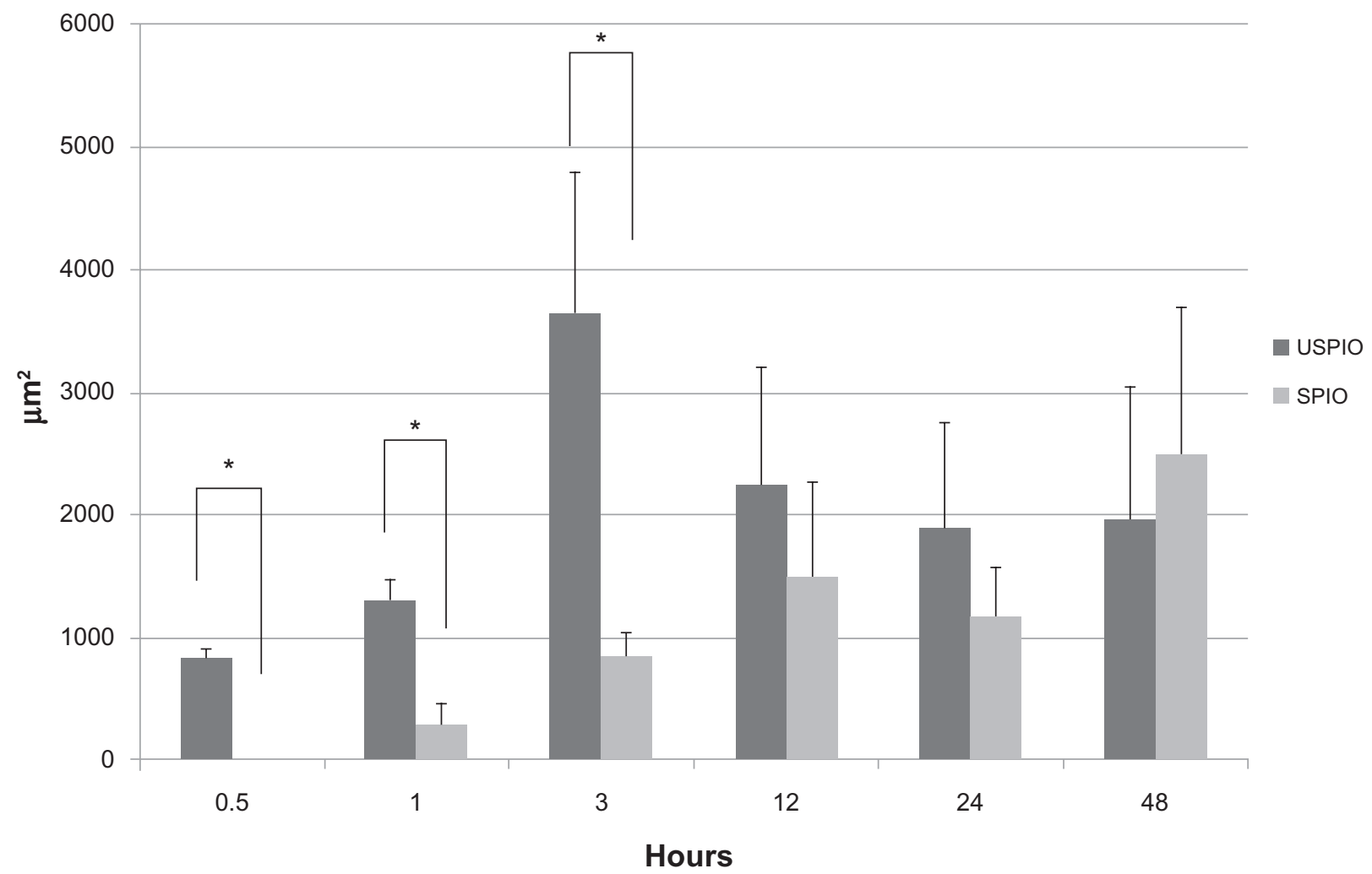

Figure 2 Comparison of Prussian-blue-stained areas $\left(\mu \mathrm{m}^{2}\right)$ in the liver. At 30 minutes, I, and 3 hours after intraperitoneal injection, iron-positive areas were significantly larger in mice treated with ultrasmall superparamagnetic iron oxide (USPIO) than with small superparamagnetic iron oxide (SPIO). Note: $* P<0.05$. 


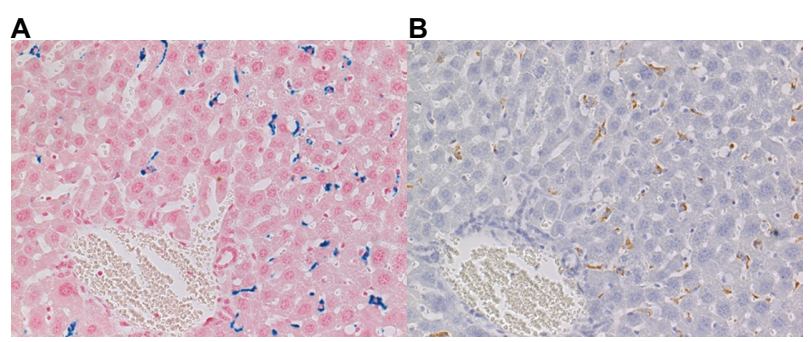

Figure 3 Histological study of the liver of mice sacrificed 48 hours after the intraperitoneal administration of ultrasmall superparamagnetic iron oxide. (A) Prussian-blue and (B) F4/80 stain (magnification $\times 200$ ). Iron-positive areas were located at the hepatic sinusoid corresponding to the area harboring Kupffer cells and were consistent with areas positive for immunohistochemical staining.

the airway and their number was increased dramatically at 3 hours postinjection and decreased thereafter, as shown in Figure 4. At all time points, iron positive areas in the lung parenchyma were larger in the mice injected with USPIO than those injected with SPIO (65 vs 29 at 30 minutes, 149 vs 52 at 1 hour, 4812 vs 1364 at 3 hours, 906 vs 201 at 12 hours, 616 vs 167 at 24 hours, and 288 vs 249 at 48 hours), as shown in Figure 5. In addition, at 30 minutes, 1, 3, and 24 hours the iron-positive areas were significantly larger in mice injected with USPIO $(P<0.05)$.

Both USPIO and SPIO were abundant at all time points in mediastinal lymph nodes; there was no difference between the two groups of mice $(P>0.05)$, as shown in Figures 6 and 7 . As in liver specimens, iron-positive areas coincided with macrophages in lung and lymph node samples.
There was no significant accumulation in the heart, great vessels, kidneys, or gastrointestinal tract. As the controls harbored abundant stores of iron, visualized as Prussianblue-positive areas, macroscopically it was observed that there was almost no difference between them and USPIOor SPIO-treated mice with respect to the spleen. Control mice manifested no significant iron deposits in organs other than the spleen.

\section{In vitro study}

While almost all cultured J774.1 cells phagocytized USPIO and SPIO, the amount of intracellular iron measured by atomic absorption photometry was significantly higher in cells treated with SPIO than with USPIO (695 vs 108 pg/cell, $P<0.05$ ), as shown in Figure 8.

\section{Discussion}

To our knowledge, this is the first study comparing the biodynamics of intraperitoneally injected USPIO and SPIO. SPIO (Resovist) is the contrast agent used in MRI and generally its safety in the clinical setting has already been established. USPIO used in this study is different in particle size from that of SPIO, but very similar in composition. Ferumoxtran-10, similar to the USPIO used in this study, was well tolerated in phase I and II studies in adult humans..$^{20,21}$ In addition, in a safety study conducted in adult rabbits, no inflammation or fibrosis occurred after intraperitoneal (IP) injection of
A

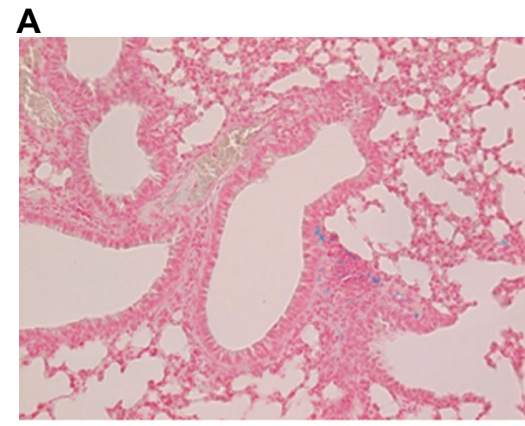

D

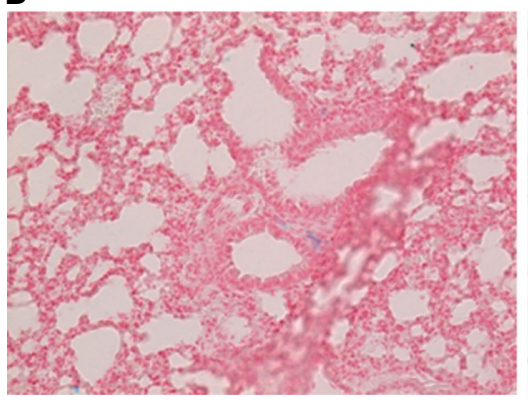

B

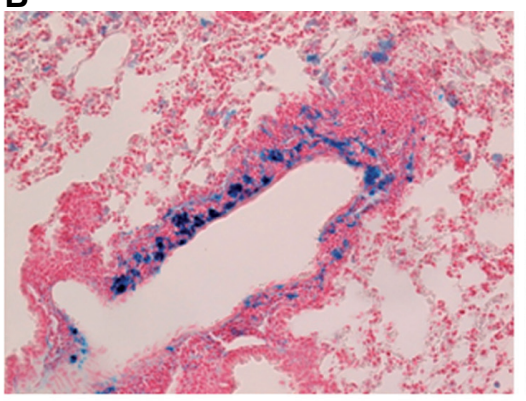

E

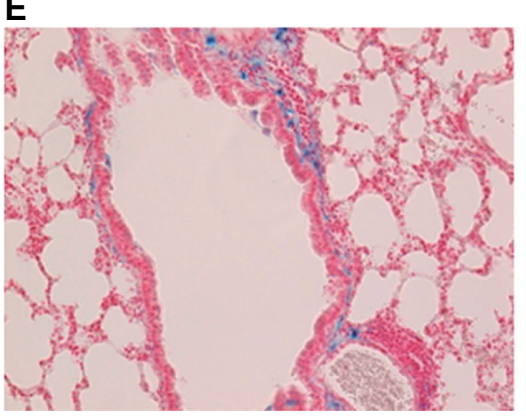

C

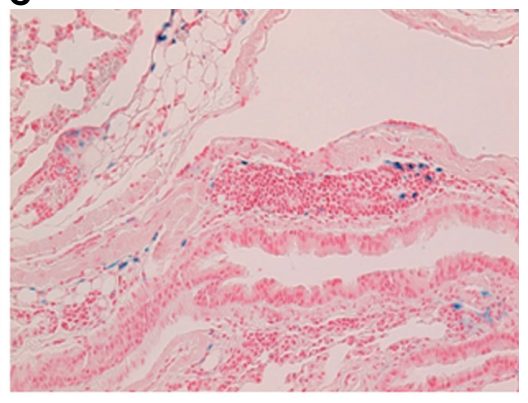

$\mathbf{F}$

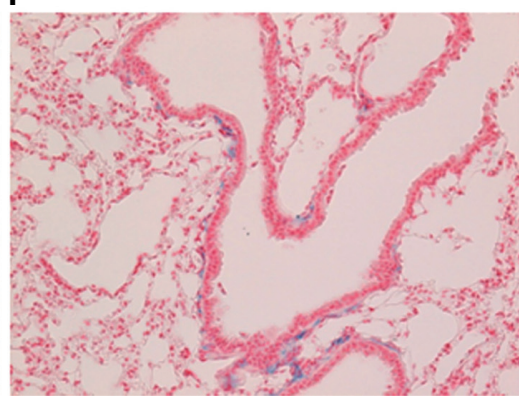

Figure 4 Prussian-blue staining of lung parenchyma around the airway (magnification $\times 200)$. (A), (B), (C): 30 minutes, 3 , and 48 hours, respectively, after intraperitoneal (IP) injection of ultrasmall superparamagnetic iron oxide. (D), (E), (F): 30 minutes, 3, and 48 hours, respectively, after IP injection of small superparamagnetic iron oxide. 


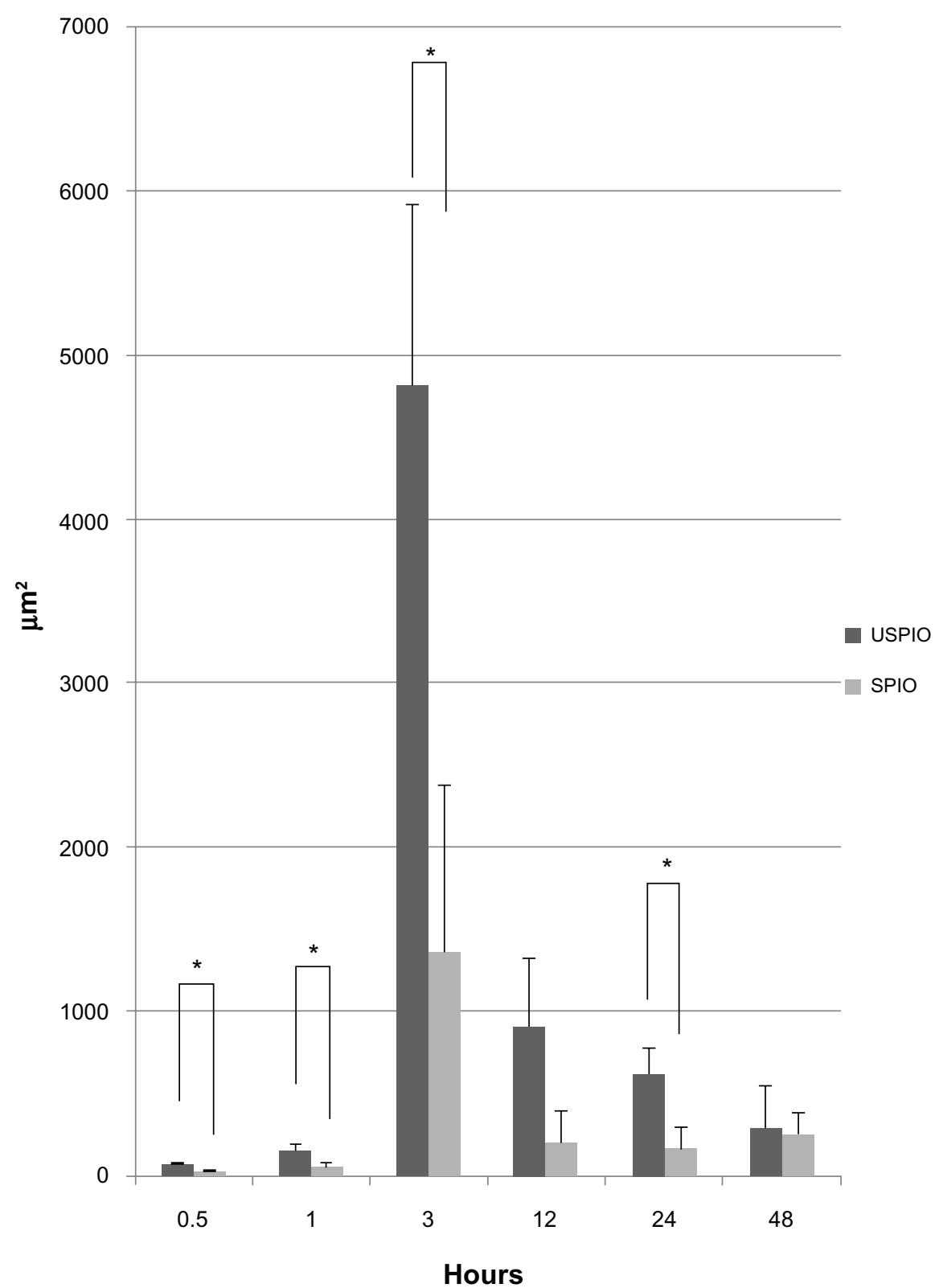

Figure 5 Comparison of Prussian-blue-stained areas $\left(\mu \mathrm{m}^{2}\right)$ in the lung parenchyma. The iron-positive areas were significantly larger in mice intraperitoneally injected with ultrasmall superparamagnetic iron oxide (USPIO) than with small superparamagnetic iron oxide (SPIO) at 30 minutes, I, 3, and 24 hours.

Note: $* P<0.05$.

NC100150, a USPIO similar to ferumoxtran-10. ${ }^{22}$ IP irondextran supplementation is known to be effective and safe in correcting iron deficiency in rats and humans. ${ }^{23,24}$ In our study, IP injection was safe and never caused the death of any mouse.

USPIO was distributed to the liver as early as 30 minutes postinjection; it peaked at 3 hours and decreased thereafter. SPIO appeared later and its distribution throughout the liver increased over time. At 12 hours postinjection, the hepatic distribution of both agents was comparable. A greater amount of USPIO than SPIO tended to be distributed to the lung parenchyma around the airway, possibly because USPIO was presented more easily to the small lymphatic vessels around the airway. There was no significant difference in lymph node accumulation between the two groups of mice.

The early distribution of intraperitoneally injected USPIO to the liver and the absence of significant differences in the lymph node distribution of USPIO and SPIO are primarily attributable to differences in the absorption route. The trapping of intravenously administered USPIO by reticuloendothelial cells in the liver and spleen has 
A

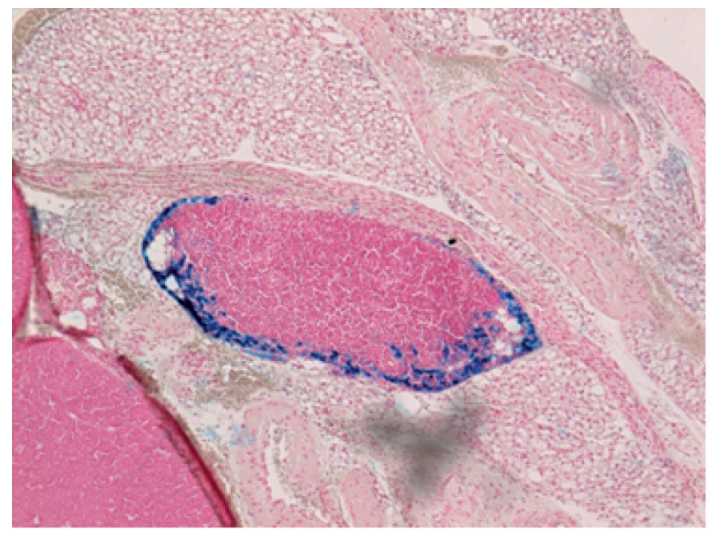

C

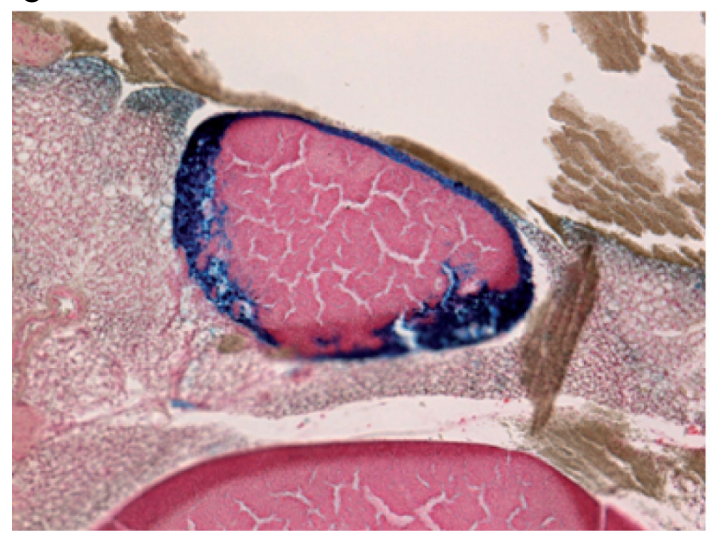

B

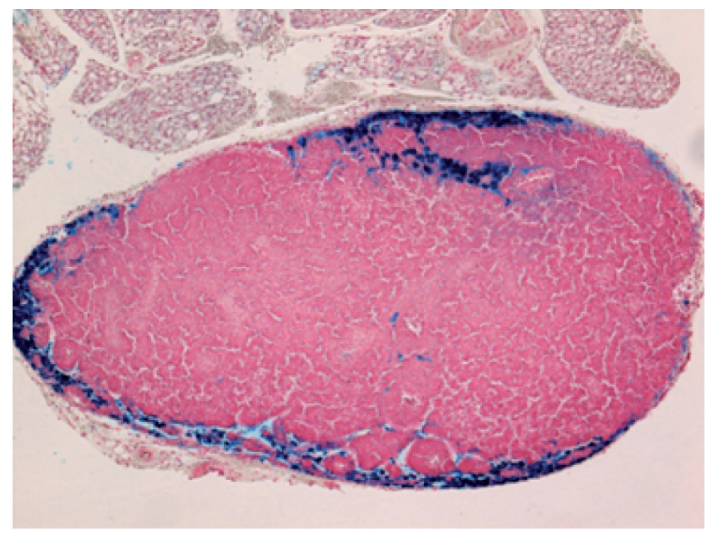

D

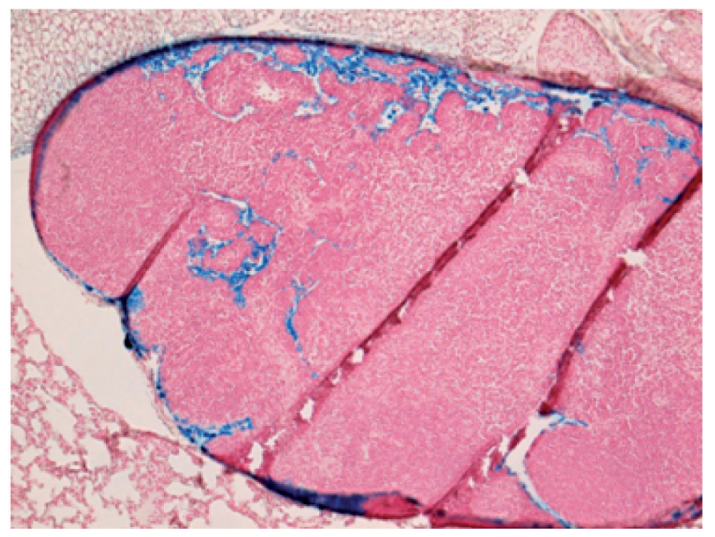

Figure 6 Prussian-blue staining of mediastinal lymph nodes (magnification $\times 100)$. (A), (B): 30 minutes and I hour after intraperitoneal (IP) injection of ultrasmall superparamagnetic iron oxide. (C), (D): 30 minutes and I hour after IP injection of small superparamagnetic iron oxide.

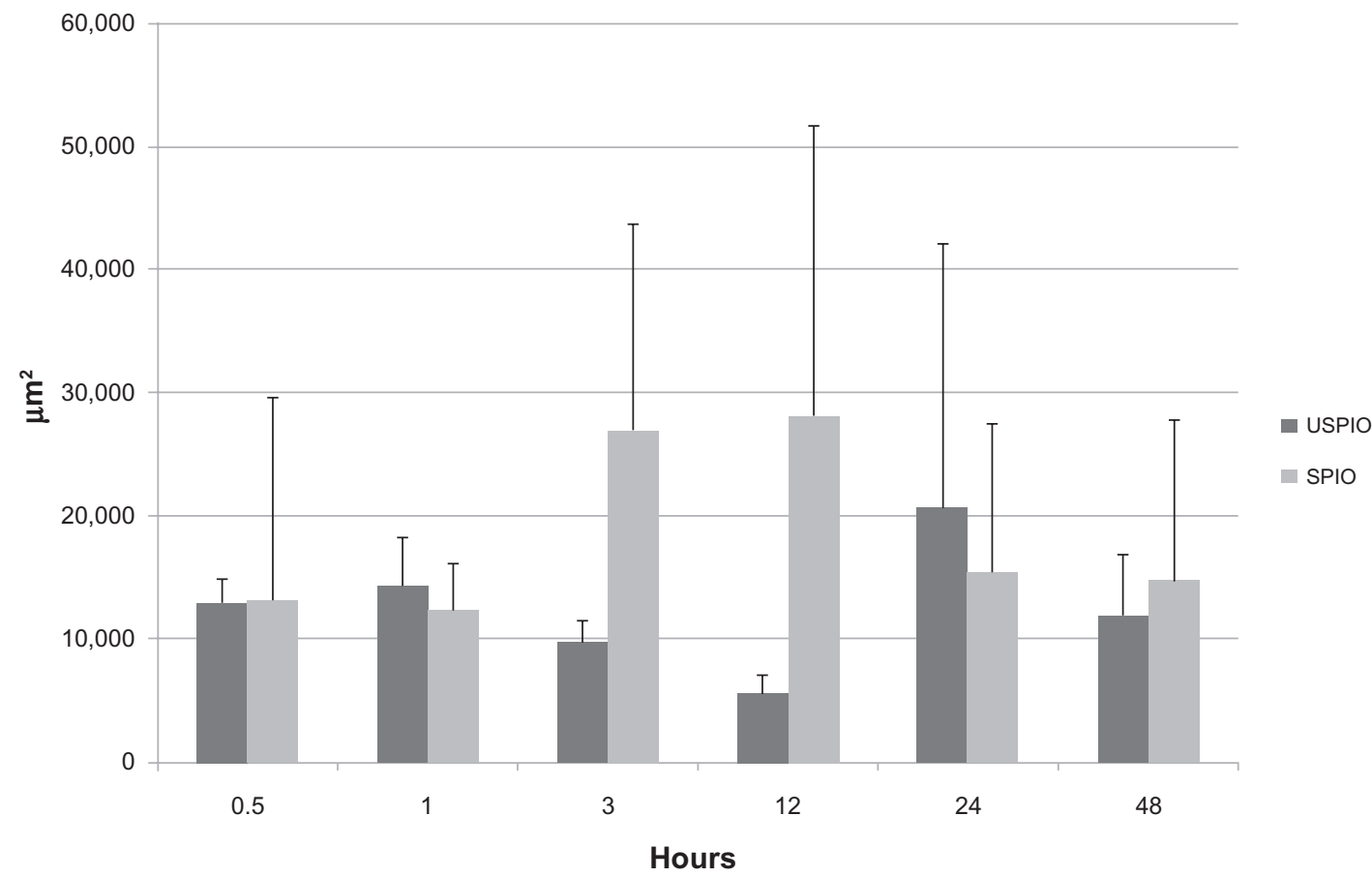

Figure 7 Comparison of distribution of ultrasmall superparamagnetic iron oxide (USPIO) and small superparamagnetic iron oxide (SPIO) in the lymph nodes. Note: $P>0.05$ at all time points. 


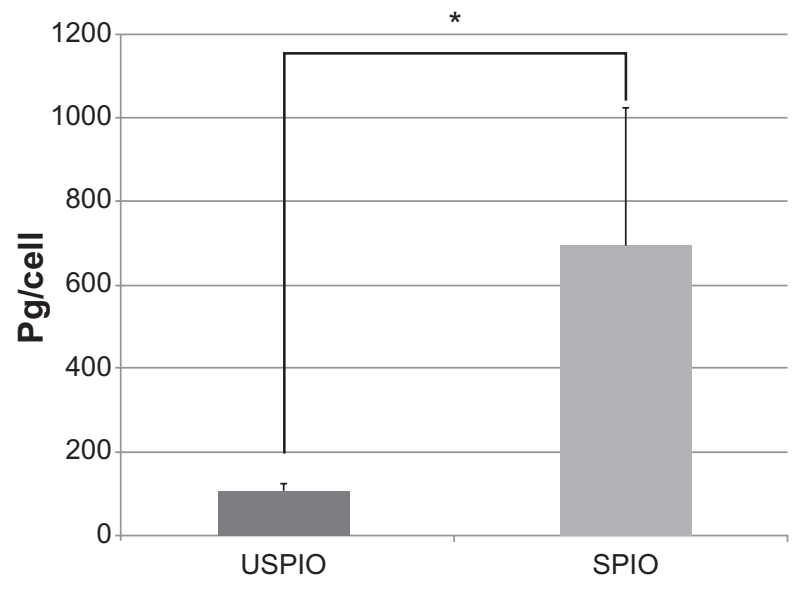

Figure 8 Comparison of the amount of intracellular iron measured by atomic absorption photometry. The amount of intracellular iron was significantly higher in cells treated with small superparamagnetic iron oxide (SPIO) than with ultrasmall superparamagnetic iron oxide (USPIO).

Note: $* P<0.05$

been reported to be difficult because the particle diameter is small and the particles persist in the blood. ${ }^{11,12}$ Lukas et $\mathrm{l}^{25}$ who studied the absorption route of intraperitoneally administered compounds, suggested that particles absorbed by the subperitoneal capillary system reached the liver via the hepatic portal system. The earlier hepatic distribution of USPIO suggests that it is delivered via this pathway. Intercellular spaces directly open to lymphatic vessels are present in the mesothelial layer of the peritoneum. The peritoneal cavity is the origin of two major lymphatic networks: the thoracic duct drains the retroperitoneal region and enters the left subclavian vein; the right lymphatic duct collects lymph from parasternal and intercostal-paravertebral lymph ducts and drains mainly through the diaphragm and, ultimately, into the right subclavian vein. In the former network, lymph passes through iliac, renal, splenic, posterior gastric, portal, superior mesenteric, and cisternal lymph nodes; in the latter network it passes through more distal (eg, parathymic) and left and right posterior mediastinal lymph nodes. ${ }^{26,27}$ The authors posited that there was not only USPIO present in abundant amounts in the lymphatic tracts due to these networks but also SPIO, which tended to be trapped in the liver with only slight distribution to the lymph nodes.

Cultured macrophages absorbed SPIO more readily than USPIO. Under conditions where the same number of macrophages respond to iron oxide compounds containing the same amount of iron, larger particles are recognized more easily. This is consistent with the hypothesis that upon intravenous (IV) infusion, reticuloendothelial cells in the liver and spleen trap SPIO more readily than USPIO.
This study had some limitations. First, the number of mice was too small for an accurate elucidation of the pharmacokinetics and prevented the exclusion of interindividual differences. Second, it could not be confirmed that the minute amounts of USPIO and SPIO used were injected accurately into the peritoneal cavity. In some instances, the agent might have been injected subcutaneously or into the bowel. Third, due to technical challenges, the pharmacokinetics of intravenously delivered USPIO or SPIO was not studied. However, considering the mechanisms by which USPIO reached the liver earlier than SPIO and the result that similar amounts of USPIO and SPIO were distributed throughout the lymph nodes, it is important to compare the differences of distribution between USPIO and SPIO via two different injection routes (IP and IV).

The findings suggest that the IP delivery of both USPIO and SPIO might be useful as a contrast agent for the study of lymph nodes in a clinical setting. The IP delivery of USPIO might also be used as a contrast agent for studying the liver. As IP delivery is not a popular injection route in clinical settings, drugs or contrast agents are currently intraperitoneally injected in limited cases of patients with cancer, peritonitis, or chronic renal failure requiring continuous ambulatory peritoneal dialysis (CAPD) ${ }^{24,28-30}$ Although clinical application is limited, the authors suggest, for cases of patients with ascites of unknown origin, that intraperitoneally injected iron particles may be very useful for getting Kupffer images of the liver and lymph node images to help diagnose metastases in these areas. Also, in cases of iron-deficient CAPD patients, intraperitoneally injected iron dextran has potential for filling both roles, as a route of entry in iron therapy and as a contrast agent for the liver and lymph nodes. In addition, depending on the diameter of the administered particles, it may be possible to deliver agents other than USPIO and SPIO intraperitoneally for the study of various organs.

\section{Conclusion}

This study suggests that intraperitoneally injected USPIO could be used more quickly than SPIO can to make Kupffer images of the liver and that both agents could help get lymph node images of similar quality.

\section{Disclosure}

The authors report no conflicts of interest in this work.

\section{References}

1. Saini S, Stark DD, Hahn PF, Wittenberg J, et al. Ferrite particles: a superparamagnetic MR contrast agent for the reticuloendothelial system. Radiology. 1987;162(1 Pt 1):211-216. 
2. Weissleder R, Stark DD, Engelstad BL, et al. Superparamagnetic iron oxide: pharmacokinetics and toxicity. AJRAm J Roentgenol. 1989;152(1): 167-173.

3. Weissleder R, Elizondo G, Wittenberg J, et al. Ultrasmall superparamagnetic iron oxide: an intravenous contrast agent for assessing lymph nodes with MR imaging. Radiology. 1990;175(2):494-498.

4. Will O, Purkayastha S, Chan C, et al. Diagnostic precision of nanoparticle-enhanced MRI for lymph-node metastases: a meta-analysis. Lancet Oncol. 2006;7(1):52-60.

5. Sigal R, Vogl T, Casselman J, et al. Lymph node metastases from head and neck squamous cell carcinoma: MR imaging with ultrasmall superparamagnetic iron oxide particles (Sinerem MR): results of a phase-III multicenter clinical trial. Eur Radiol. 2002;12(5): 1104-1113.

6. Daldrup HE, Link TM, Blasius S, et al. Monitoring radiation-induced changes in bone marrow histopathology with ultra-small superparamagnetic iron oxide (USPIO)-enhanced MRI. J Magn Reson Imaging. 1999;9(5):643-652.

7. Senéterre E, Weissleder R, Jaramillo D, et al. Bone marrow: ultrasmall superparamagnetic iron oxide for MR imaging. Radiology. 1991; 179(2):529-533

8. Harisinghani MG, Saini S, Weissleder R, et al. Splenic imaging with ultrasmall superparamagnetic iron oxide ferumoxtran-10 (AMI-7227): preliminary observations. J Comput Assist Tomogr. 2001;25(5): $770-776$.

9. Weissleder R, Hahn PF, Stark DD, et al. Superparamagnetic iron oxide: enhanced detection of focal splenic tumors with MR imaging. Radiology. 1988;169(2):399-403.

10. Stark DD, Weissleder R, Elizondo G, et al. Superparamagnetic iron oxide: clinical application as a contrast agent for MR imaging of the liver. Radiology. 1988;168(2):297-301.

11. Weissleder R, Elizondo G, Wittenberg J, et al. Ultrasmall superparamagnetic iron oxide: characterization of a new class of contrast agents for MR imaging. Radiology. 1990;175(2):489-493.

12. Vassallo P, Matei C, Heston WD, et al. AMI-227-enhanced MR lymphography: usefulness for differentiating reactive from tumor-bearing lymph nodes. Radiology. November 1994;193(2):501-506.

13. Anzai Y, Prince MR, Chenevert TL, et al. MR angiography with an ultrasmall superparamagnetic iron oxide blood pool agent. J Magn Reson Imaging. 1997;7(1):209-214.

14. Loubeyre P, Zhao S, Canet E, et al. Ultrasmall superparamagnetic iron oxide particles (AMI 227) as a blood pool contrast agent for MR angiography: experimental study in rabbits. J Magn Reson Imaging. 1997;7(6):958-962.

15. Renkin EM. Multiple pathways of capillary permeability. Circ Res. 1977;41(6):735-743.
16. Banerjee S, Narayanan K, Mizutani T, et al. Murine coronavirus replication-induced p38 mitogen-activated protein kinase activation promotes interleukin-6 production and virus replication in cultured cells. J Virol. 2002;76(12):5937-5948.

17. Khoury M, Escriou V, Courties G, et al. Efficient suppression of murine arthritis by combined anticytokine small interfering RNA lipoplexes. Arthritis Rheum. 2008;58(8):2356-2367.

18. Taniguchi S, Yanase T, Kobayashi K, et al. Dehydroepiandrosterone markedly inhibits the accumulation of cholesteryl ester in mouse macrophage J774-771 cells. Atherosclerosis. 1996;126(1):143-154.

19. Jo J, Aoki I, Tabata Y. Design of iron oxide nanoparticles with different sizes and surface charges for simple and efficient labeling of mesenchymal stem cells. J Control Release. 2010;142(3):465-473.

20. Sharma R, Saini S, Ros PR, et al. Safety profile of ultrasmall superparamagnetic iron oxide ferumoxtran-10: phase II clinical trial data. J Magn Reson Imaging. 1999;9(2):291-294.

21. McLachlan SJ, Morris MR, Lucas MA, et al. Phase I clinical evaluation of a new iron oxide MR contrast agent. J Magn Reson Imaging. 1994;4(3):301-307.

22. Hilfiker PR, Debatin JF, Tarlo K, et al. Assessment of peritoneal tolerance of a new MR blood pool contrast agent in rabbits. Invest Radiol. 1999;34(11):722-727.

23. Reddy DK, Moore HL, Lee JH, et al. Chronic peritoneal dialysis in iron-deficient rats with solutions containing iron dextran. Kidney Int. 2001;59(2):764-773.

24. Bastani B, Galley S. Intraperitoneal iron-dextran as a potential route of iron therapy in CAPD patients. Perit Dial Int. 1996;16(6):646-648.

25. Lukas G, Brindle SD, Greengard P. The route of absorption of intraperitoneally administered compounds. J Pharmacol Exp Ther. 1971; 178(3):562-564.

26. Rusznyák L, Földi M, Szabo G. Filtration and absorption through serous membranes. Lymphatics and Lymph Circulation. 2nd ed. Oxford: Pergamon Press; 1967:478-497.

27. Tilney NL. Patterns of lymphatic drainage in the adult laboratory rat. J Anat. 1971;109(Pt 3):369-383.

28. Zylberberg B, Dormont D, Antoine JM, et al. First line immunochemotherapy with cisplatin-based protocol by intraperitoneal and intravenous routes in ovarian cancer: technique and results of 82 cases. Eur J Obstet Gynecol Reprod Biol. 1996;66(1):57-64.

29. Ruf P, Kluge M, Jäger M, et al. Pharmacokinetics, immunogenicity and bioactivity of the therapeutic antibody catumaxomab intraperitoneally administered to cancer patients. Br J Clin Pharmacol. 2010; 69(6):617-625.

30. Tamano M, Hashimoto T, Kojima K, et al. Diagnosis of hepatic hydrothorax using contrast-enhanced ultrasonography with intraperitoneal injection of Sonazoid. J Gastroenterol Hepatol. 2010;25(2):383-386.
International Journal of Nanomedicine

\section{Publish your work in this journal}

The International Journal of Nanomedicine is an international, peerreviewed journal focusing on the application of nanotechnology in diagnostics, therapeutics, and drug delivery systems throughout the biomedical field. This journal is indexed on PubMed Central, MedLine, CAS, SciSearch $\AA$, Current Contents ${ }^{\circledR} /$ Clinical Medicine,

\section{Dovepress}

Journal Citation Reports/Science Edition, EMBase, Scopus and the Elsevier Bibliographic databases. The manuscript management system is completely online and includes a very quick and fair peer-review system, which is all easy to use. Visit http://www.dovepress.com/ testimonials.php to read real quotes from published authors. 